Academic Platform Journal of Engineering and Science

journal homepage: $\underline{\text { http://apjes.com/ }}$

\title{
Design Equation for Operating Frequency of Patch Antenna with a Rectangular Tuning Stub at Early Phase 5G Bands
}

\author{
*11Barış Gürcan Hakanoğlu, ${ }^{2}$ Şekip Esat Hayber, ${ }^{3}$ Mustafa Türkmen \\ ${ }^{1}$ Kırşehir Ahi Evran University, Department of Electronics and Automation, 40300, Kaman, Kırşehir, \\ bghakanoglu@ahievran.edu.tr, (1) \\ ${ }^{2}$ Kırşehir Ahi Evran University, Department of Electrical and Electronics Engineering, 40100, Bağbaşı, Kırşehir, \\ sehayber@ahievran.edu.tr, (D) \\ ${ }^{3}$ Erciyes University, Department of Electrical and Electronics Engineering, 38039, Talas, Kayseri, \\ turkmen@erciyes.edu.tr,
}

Research Paper

Arrival Date: 05.07.2020

Accepted Date: 22.08 .2021

\begin{abstract}
In this paper, microstrip patch antennas with rectangular tuning stubs are analyzed, and variations of the resonant frequency depending on the dimensions of the stub have been studied. According to these variations, a design equation has also been proposed for the antennas. The operating frequencies are selected from the early phase 5G bands (700, 2300, 3500, and 4700 $\mathrm{MHz}$ ), but the equation validity is tested for additional frequencies as $6500 \mathrm{MHz}$ and $8500 \mathrm{MHz}$ which are randomly selected. To identify the material effects, analyses are conducted for two different dielectrics, FR4 and Rogers RT/Duroid 5880. The calculations have been performed for twelve different reference antennas and twelve stub-placed modified antennas. It has been shown that the patch antennas with rectangular tuning stubs pave the way to tune the antenna resonance up to $7.5 \%-27.6 \%$ of its reference design frequency. For example, the proposed structure designed for $700 \mathrm{MHz}$ can shift the resonant frequency up to $511.5 \mathrm{MHz}$ for FR4 and $507 \mathrm{MHz}$ for Rogers RT/Duroid 5880. Moreover, the shifting in frequency at $8500 \mathrm{MHz}$ can reach 7950 $\mathrm{MHz}$ for FR4 and $7865 \mathrm{MHz}$ for Rogers RT/Duroid 5880 with reasonable $S_{1 l}$ levels.
\end{abstract}

Keywords: Microstrip Antenna, Rectangular Patch Antenna, Early 5G, Tuning Stub, Design Equation.

\section{INTRODUCTION}

Microstrip patch antennas have many advantages, such as being lightweight, cost-effective, and having compatible dimensions with various surfaces, circuits, and devices. However, the major shortcoming of such antennas is their limited bandwidth which makes the input impedance very fragile to fabrication errors and dielectric material tolerances. So, causing changes in the effective length of the patch, manual tuning can adjust the operating frequency. It has been shown that the resonant frequency of a patch antenna can be fine-tuned by placing a tuning stub on one edge of the antenna with the tuning range dependent on the length and the width of the stub [1]. Tunable frequency microstrip antennas can also be realized using RF microelectromechanical systems (MEMS) technology [2]. Tuning and miniaturizing can be performed simultaneously by etching slots in the patch and using thin posts placed near the edge [3]. Besides, it has been demonstrated that a microstrip patch antenna can be tuned on a liquid crystal substrate using a DC bias. The simulated and measured tuning ranges have been found at $8 \%$ and $4 \%$, respectively [4]. A microstrip patch antenna on a tunable electromagnetic band-gap (EBG) structure is proposed with the feature of performing the tuning through a diode-loaded EBG substrate [5]. Another application area for tuning stubs is to obtain triple frequency operation and circular polarization $[6,7]$. Moreover, stubs are proposed to enhance the patch's bandwidth and make the whole structure more compact $[8$, 9].

The motivation for the design frequencies of the antennas in this paper is the early phase $5 \mathrm{G}$ bands. Three regions, such as the low range up to $3 \mathrm{GHz}$, mid-range between $3 \mathrm{GHz}-$ $6 \mathrm{GHz}$, and the high range above $6 \mathrm{GHz}$, are studied [10]. These bands are still under consideration for some countries, but some have already completed the frequency assignments. Many countries in Europe decided to utilize the 3400-3800 $\mathrm{MHz}$ range for the early phase of $5 \mathrm{G}$. The United States launches out 3550-3700 MHz band for a new Citizens Broadband Radio Service to accommodate various commercial services on a shared basis. As a different part of the spectrum, the $4400-5000 \mathrm{MHz}$ band is concerned by Japan and China. Japan has assigned 4400-4900 MHz band for 5G trials, and China defined 4800-5000 MHz band for $5 \mathrm{G}$ [11]. Besides, the $2300 \mathrm{MHz}$ band is also released to be

${ }^{* 1}$ Corresponding author: Kırşehir Ahi Evran University, Department of Electronics and Automation, 40300, Kırşehir, Turkey

bghakanoglu@ahievran.edu.tr 
a candidate for $5 \mathrm{G}$ communication in Sweden and United Kingdom [12]. Moreover, the Radio Spectrum Policy Group of the European Commission reveals that the $700 \mathrm{MHz}$ band also needs to be operated in practice to make possible nationwide and indoor 5G coverage [13].

This study performs a detailed parametric analysis of the rectangular tuning stub (RTS) effect on the resonant frequency of microstrip patch antenna (MPA) and formulates a design equation. Microstrip patch antenna with rectangular tuning stub will be called MPA-RTS throughout the paper. RTS is placed on the top edge of the patch. The variations of stub edge dimensions result in different ways on the resonant frequencies and reflection coefficient levels. While the width of the stub variation causes a slight shift in resonant frequency and not so much change in reflection coefficient levels, the length of the stub variation produces a significant change in frequency shifting and reflection coefficient levels.

Moreover, the amount of frequency shifting varies according to the operating band. While an MPA-RTS is operating at $700 \mathrm{MHz}$ shifts the resonant frequency up to $511.5 \mathrm{MHz}$ for FR4 and $507 \mathrm{MHz}$ for Rogers RT/Duroid 5880, an 8500 $\mathrm{MHz}$ operating MPA-RTS shifts the resonant frequency up to $7950 \mathrm{MHz}$ for FR4 and $7865 \mathrm{MHz}$ for Rogers RT/Duroid 5880 with reasonable return loss levels.

\section{DESIGN}

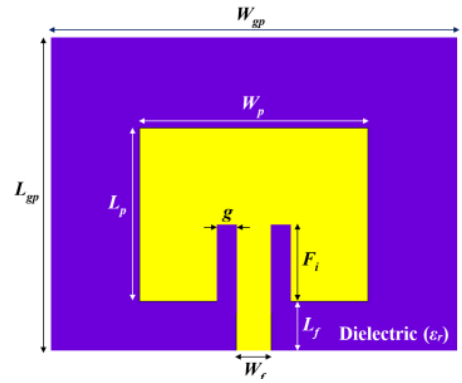

a

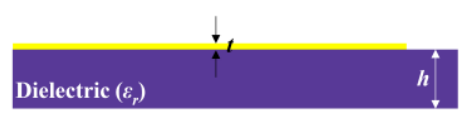

b

Figure 1. a. Top view of MPA b. Side view of MPA.

The reference antennas are designed for early phase $5 \mathrm{G}$ and frequencies outside the $5 \mathrm{G}$ bands using the proposed Equations 1-4 derived from the patch antenna's transmission line model [14]. Also, to observe the material effects, two different dielectrics with different heights are used for the substrates, FR4 and Rogers RT/Duroid 5880, with the permittivity, $\varepsilon_{r}$ of 4.4 and 2.2, respectively. The heights of the substrates are $1.5 \mathrm{~mm}$ for FR4 and $0.787 \mathrm{~mm}$ for RT5880. Figure 1a shows the top view of an example design for the antenna, and Figure $1 \mathrm{~b}$ shows the side view of the structure. All antennas are designed with the same procedure. First, with Equation 1, the width of the patch, $W_{p}$ is calculated based on the following parameters: $f_{r}, \varepsilon_{r}$, and $h$, where $f_{r}$ is the resonant frequency, $\varepsilon_{r}$ is the relative permittivity, and $h$ is the substrate height. After that, Equation 2 enables us to obtain the effective permittivity, $\varepsilon_{\text {reff, which should be known }}$ to calculate the length extension, $\Delta L_{p}$ due to the fringing field with the condition of $W_{p} / h>1$ (Equation 3). Finally, with Equation 4 , the patch length, $L_{p}$, can be calculated.

$$
\begin{gathered}
W_{p}=\frac{v_{0}}{2 f_{r}} \sqrt{\frac{2}{\varepsilon_{r}+1}} \\
\varepsilon_{e f f}=\frac{\varepsilon_{r}+1}{2}+\frac{\varepsilon_{r}-1}{2}\left(1+12 \frac{h}{W_{p}}\right)^{-\frac{1}{2}} \\
\frac{\Delta L_{p}}{h}=0.412 \frac{\left(\varepsilon_{e f f}+0.3\right)\left(\frac{W_{p}}{h}+0.264\right)}{\left(\varepsilon_{e f f}-0.258\right)\left(\frac{W_{p}}{h}+0.8\right)} \\
L_{p}=\frac{v_{0}}{2 f_{r} \sqrt{\varepsilon_{e f f}}}-2 \Delta L_{p}
\end{gathered}
$$

There are no specific rules for the dielectric substrate dimensions. It is recommended that the length of the substrate, $L_{g p}$, and the width of the substrate, $W_{g p}$, should be greater than $6 h+L_{p}$ and $6 h+W_{p}$, respectively [15]. We performed a parametric analysis and an optimization about the edge dimensions of the substrate and took the values at which we obtain the reasonable $S_{11}$ levels. The radiating part is modeled as a lossy conductor, and its thickness, $t$, is set to $0.035 \mathrm{~mm}$ (Figure 1b). The feeding is performed through a microstrip feed line with a width of $W_{f}$ and the length of $L_{f}$ together with an inset feeding part with the dimensions of the length $F_{i}$ and the width $g$. For each design, $W_{f}$ is taken as 3 $\mathrm{mm}$ for fabrication and impedance matching conditions for 50 ohms SMA (SubMiniature version A) connector. Due to the same reason, the width of the inset feeding, $g$, is calculated together with $W_{f}, h$, and $\varepsilon_{r}$ to match $50 \mathrm{ohms}$. The length of inset feeding, $F_{i}$, is obtained through parametric analyses until we have the lowest $S_{11}$ levels. The optimized parameters of the MPAs are summarized in Tables 1 and 2. 
Table 1. The optimized parameters of the MPAs for FR4 ( $\left.\varepsilon_{r}=4.4\right), h=1.5 \mathrm{~mm}$.

\begin{tabular}{ccccccc}
\hline \multirow{2}{*}{$\begin{array}{c}\text { Parameters } \\
(\mathrm{mm})\end{array}$} & 0.7 & 2.3 & 3.5 & 4.7 & 6.5 & 8.5 \\
\cline { 2 - 7 } & 233.66 & 71.81 & 47.46 & 34.81 & 25.83 & 19.85 \\
\hline$W_{g p}$ & 182.90 & 55.75 & 36.57 & 26.80 & 19.49 & 14.75 \\
$L_{g p}$ & 130.32 & 39.66 & 26.06 & 19.41 & 14.03 & 10.73 \\
$W_{p}$ & 101.95 & 30.76 & 20.02 & 14.74 & 10.44 & 7.78 \\
$L_{p}$ & 35.30 & 11.70 & 8.20 & 6.40 & 4.60 & 3.50 \\
$F_{i}$ & 0.68 & 0.21 & 0.14 & 0.11 & 0.08 & 0.06 \\
$g$ & 38.89 & 11.84 & 7.78 & 5.79 & 4.19 & 3.20 \\
$L_{f}$ & 3.00 & 3.00 & 3.00 & 3.00 & 3.00 & 3.00 \\
$W_{f}$ & & & & &
\end{tabular}

Table 2. The optimized parameters of the MPAs for Rogers RT/Duroid $5880\left(\varepsilon_{r}=2.2\right), h=0.787 \mathrm{~mm}$.

\begin{tabular}{ccccccc}
\hline \multirow{2}{*}{$\begin{array}{c}\text { Parameters } \\
(\mathrm{mm})\end{array}$} & 0.7 & 2.3 & 3.5 & 4.7 & 6.5 & 8.5 \\
\cline { 2 - 7 } & 314.19 & 95.95 & 63.20 & 47.16 & 34.19 & 26.22 \\
\hline$W_{g p}$ & 255.41 & 77.65 & 50.96 & 37.88 & 27.31 & 20.79 \\
$L_{g p}$ & 169.05 & 51.52 & 33.86 & 25.21 & 18.23 & 13.94 \\
$W_{p}$ & 144.07 & 43.60 & 28.51 & 21.12 & 15.14 & 11.45 \\
$L_{p}$ & 35.30 & 16.20 & 11.10 & 8.60 & 6.20 & 4.80 \\
$F_{i}$ & 0.95 & 0.29 & 0.19 & 0.14 & 0.11 & 0.08 \\
$g$ & 38.89 & 11.83 & 7.78 & 5.79 & 4.19 & 3.20 \\
$L_{f}$ & 3.00 & 3.00 & 3.00 & 3.00 & 3.00 & 3.00 \\
$W_{f}$ & & & & &
\end{tabular}

According to the above results, reference antennas are modeled using Computer Simulation Technology Microwave Studio (CST MWS) [16]. To tune the resonant frequency, an RTS has been placed on the top edge of the patch with the dimensions of $L_{R T S}$ for the length and $W_{R T S}$ for the width. Figure 2 shows an example model for MPA-RTS. This figure shows that with the help of the RTS, it is possible to design antennas occupying less room. The dimensions of the RTS affect the resonant frequency and reflection coefficient levels of the antennas. So, a detailed parametric analysis should be performed about $L_{R T S}$ and $W_{R T S}$.

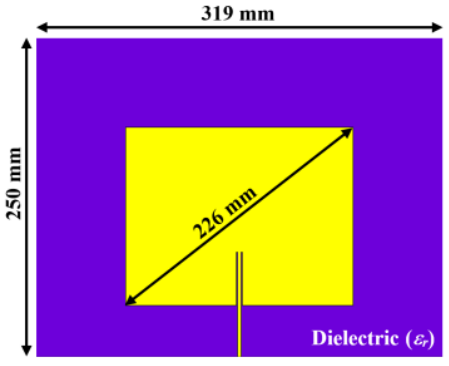

$\mathbf{a}$

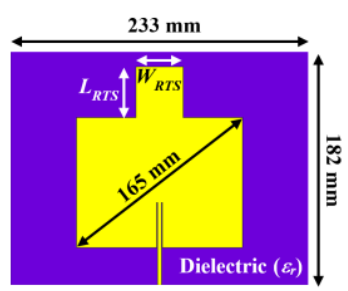

b
Figure 2. MPAs operating at $511.5 \mathrm{MHz}$ a. without RTS b. with RTS

\section{RESULTS AND DISCUSSIONS}

The effects of the variation of the stub parameters on the resonance frequency, $f_{r}$, and reflection coefficient parameters, $S_{11}$, are investigated using time-domain analysis for wideband or multiband antennas in CST MWS based on finite integration technique (FIT). For the study, FR4 material is preferred for dielectric substrate, and the results are also confirmed for a different dielectric material such as Rogers RT/Duroid5880. Other sized antennas are selected at quite different frequency values in addition to $5 \mathrm{G}$ frequencies. Thus, the obtained approximate model is valid for a wide range.

\subsection{Effect of RTS parameters}

MPA-RTS structures are obtained by adding RTSs to MPAs operating at early phase $5 \mathrm{G}$ frequencies such as 700, 2300, $3500,4700 \mathrm{MHz}$. In addition to these frequencies, antennas are also analyzed at 6500 and $8500 \mathrm{MHz}$ to confirm the validity of the obtained equation. In each case, the parametric analysis starts with $W_{R T S} \times L_{R T S}=0.5 \mathrm{~mm} \times 0.5 \mathrm{~mm}$ until they reach at the near endpoints of the patch for $W_{R T S}$ and of the substrate for the $L_{R T S}$. The variation path along the $L_{R T S}$ can be called dielectric substrate distance (DSD). $0.5 \mathrm{~mm}$ is chosen as the step size for both parameters of RTS. The results of the FIT-based time-domain analysis for wideband or multiband antennas in CST MWS obtained from 4700 $\mathrm{MHz}$ are shown in Figure 3 as an example. All of the results obtained for the other frequencies examined in this study have similar characteristics to those for $4700 \mathrm{MHz}$ which will be shown in the next section as an approach for the MPA-RTS. 

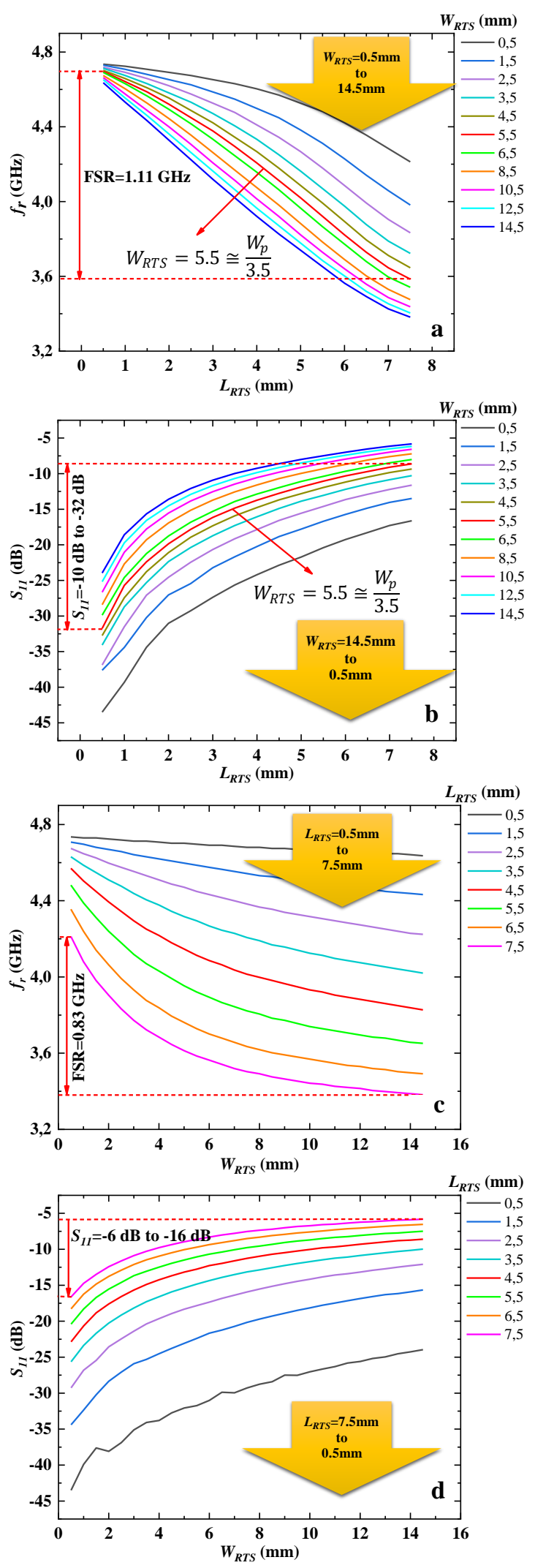

Figure 3. The effect of RTS parameters on the operating frequency and $S_{1 I}$ of the MPA-RTS a. fr versus $\mathrm{L}_{\mathrm{RTS}}$ b. $S_{1 I}$ versus $\mathrm{L}_{\mathrm{RTS}}$ c. $f_{r}$ versus $\mathrm{W}_{\mathrm{RTS}}$ d. $S_{11}$ versus $\mathrm{W}_{\mathrm{RTS}}$
Figure 3a-3d shows the variation of resonance frequencies and related $S_{I I}$ parameters when $L_{R T S}$ and $W_{R T S}$ change simultaneously along the dielectric and the width of the patch, respectively. It can be seen from Figure 3a that the increase of $L_{R T S}$ and $W_{R T S}$ result in shifting the resonances downwards, beginning from $4700 \mathrm{MHz}$ at which the antenna was initially designed. The frequency shifting range called FSR is $1110 \mathrm{MHz}$ from $4700 \mathrm{MHz}$ to $3590 \mathrm{MHz}$ in Figure $3 \mathrm{a}$, where the $S_{11}$ levels are acceptable. Figure $3 \mathrm{~b}$ shows that when $W_{R T S}$ decreases, $S_{11}$ levels increase in a negative direction up to $-32 \mathrm{~dB}$, which is desirable for these parameters. However, $S_{I l}$ levels are not at desired levels for all $W_{R T S}$ values. From Figure $3 \mathrm{a}$ and Figure $3 \mathrm{~b}$, we should find a particular value for $W_{R T S}$ at which we get a wide shifting range of frequencies and reasonable $S_{11}$ levels at the same time. From the plots, this optimal value is reached at the point when $W_{R T S}=5.5 \mathrm{~mm}$ or $1 / 3.5$ of $W_{p}$, which can also be expressed as follows,

$$
W_{R T S}=W_{p} / 3.5
$$

Figure $3 \mathrm{c}$ and Figure $3 \mathrm{~d}$ show that the effect of $\mathrm{W}_{\mathrm{RTS}}$ on the frequency and $S_{11}$ parameters for different $L_{R T S}$ values. However, both the amount of change in FSR and the values of $S_{11}$ are not at desired levels. Hence, we can say that the main effect on resonance is due to $L_{R T S}$. As a result, by placing RTS, microstrip antennas will operate at different frequencies according to variations of the length of the stub.

The effects of the variation of $L_{R T S}$ on the frequency are given in Figure 4 by taking the $W_{R T S}$ as constant for the antenna at each frequency as in Equation 5. Here, the limit value of $L_{R T S}$ is the size of the dielectric substrate. Analyzes were performed for more values by decreasing the operating frequency and thus increasing the antenna dimensions. In Figure 4, while the solid purple line shows the $L_{R T S}$ frequency change for the FR4 substrate, the light blue solid line shows the $L_{R T S}$ - frequency change for the RT5880 substrate. The change of the reflection coefficient parameters of the antenna with the increase of $L_{R T S}$ is given in the graphs as inset figures. Thus, it can be seen in which values the reflection coefficient parameters fall below $-10 \mathrm{~dB}$ due to the frequency effect of $L_{R T S}$. Then, an equation is formed with these parametric analysis results, which is explained in Section 3.2.

\subsection{An Approach for Patch Antenna with RTS}

A mathematical approach that does not require multiple simulation processes is developed in this section. When the condition of $W_{R T S}=W_{p} / 3.5$ is met, $f_{R T S} \alpha \exp \left(-0.7 L_{R T S} / W_{p}\right)$ can be derived. So, design simplicity is established. Equation 6 shows the expressions for the operating frequency of the regression analysis $\left(\mathrm{R}^{2}=0.98\right)$ on the time domain analysis for wideband or multiband antennas in CST MWS. It can also be calculated with this equation the results with different $W_{p}, L_{R T S}$, and $\varepsilon_{r}$ values from the MPA-RTS structures satisfying the condition $W_{R T S}=W_{p} / 3.5$. 


$$
\begin{array}{r}
f_{R T S}=\frac{v_{0}}{2 W_{p}} \sqrt{\frac{2}{\varepsilon_{r}+1}} \times e^{-\left(\frac{0.7 L_{R T S}}{W_{p}}\right)} \\
=f_{r} \times e^{-\left(\frac{0.7 L_{R T S}}{W_{p}}\right)}
\end{array}
$$

The lower limit of Equation 6 approaches Equation 1, which is valid for conventional MPAs. The lower limit is expressed as follows:

$$
\lim _{L_{R T S} \rightarrow 0} f_{R T S}=f_{r}
$$

Similarly, the upper boundary condition of Equation 6 tends to reduce the resonance frequency of the antenna by about $24 \%$.

$$
\lim _{L_{R T S} \rightarrow(\mathrm{DSD})} f_{R T S} \cong 0.76 \times f_{r}
$$

Moreover, percentage error values are shown in Figure 5. Percentage error values differ according to the frequency values and substrate material. In this context, according to the graphs for $f_{r}=700 \mathrm{MHz}, 2300 \mathrm{MHz}, 3500 \mathrm{MHz}$, and 4700 $\mathrm{MHz}$, the percentage error values for RT5880 are slightly higher than for FR4 in general. Similarly, according to the graphs for $6500 \mathrm{MHz}$ and $8500 \mathrm{MHz}$, it was seen that the percentage error value in FR4 is higher than in RT5880. In other words, the performance of Equation 6 is better for antennas built with RT5880 at high frequencies. It can be concluded that the error values average is $2 \%$ for the first four frequency values, and the highest value rises to $4.5 \%$ in a very narrow region. Moreover, the average error value for $6500 \mathrm{MHz}$ is $2.87 \%$, which is $3.76 \%$ for $8500 \mathrm{MHz}$. Considering all frequencies and two types of substrate materials, the most common percentage error average was calculated as $2.44 \%$.

Table 3. Comparison of similar studies in the literature with the current study.

\begin{tabular}{lllcc}
\hline \multicolumn{1}{c}{ Structure } & $\begin{array}{c}\text { Frequency } \\
\mathrm{GHz}\end{array}$ & $\begin{array}{c}\text { Tuning } \\
\text { Range }\end{array}$ & $\begin{array}{c}\text { Formed } \\
\text { Equation }\end{array}$ \\
\hline$[1] \quad \begin{array}{l}\text { Rectangular patch } \\
\text { antenna with } \\
\text { rectangular tuning } \\
\text { stubs }\end{array}$ & 2.95 & $3.33 \%$ & No \\
\hline C17] & $\begin{array}{l}\text { Circular patch } \\
\text { antenna with } \\
\text { rectangular tuning } \\
\text { stubs }\end{array}$ & 1.695 & $9 \%$ & No \\
\hline$[18]$ & $\begin{array}{l}\text { Tunable stacked } \\
\text { patch PIFA }\end{array}$ & 0.745 & $10 \%$ & No \\
\hline \multirow{5}{*}{ This work } & $\begin{array}{l}\text { Rectangular patch } \\
\text { antenna with a } \\
\text { rectangular tuning } \\
\text { stub }\end{array}$ & 0.7 & $27 \%$ & Yes \\
\cline { 2 - 5 } & $\begin{array}{l}\text { Rectangular patch } \\
\text { antenna with a } \\
\text { rectangular tuning } \\
\text { stub }\end{array}$ & 3.5 & $17 \%$ & Yes \\
\hline
\end{tabular}



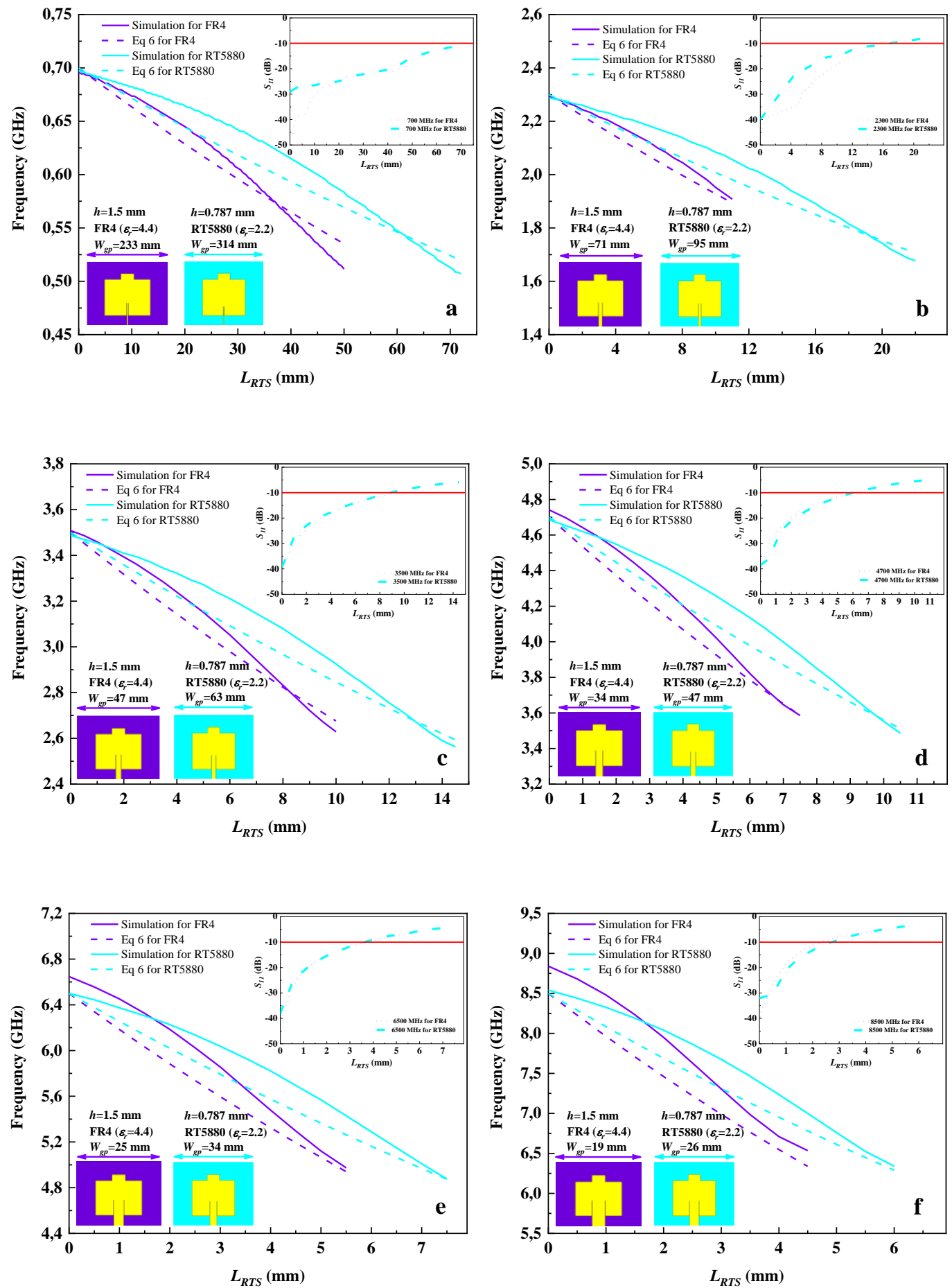

Figure 4. Comparisons of operating frequencies of MPA-RTSs with simulation and Equation 6 (Eq 6 ) for FR4 and Rogers RT/Duroid 5880 a. $f_{r}=700 \mathrm{MHz}$ b. $f_{r}=2300 \mathrm{MHz}$ c. $f_{r}=3500 \mathrm{MHz}$ d. $f_{r}=4700 \mathrm{MHz}$ e. $f_{r}=6500 \mathrm{MHz}$

f. $f_{r}=8500 \mathrm{MHz}$ effect of RTS parameters on the operating frequency and $S_{11}$ of the MPA-RTS a. $f_{r}$ versus LRTS b. $S_{11}$ versus $\mathrm{L}_{\mathrm{RTS}}$ c. $f_{r}$ versus $\mathrm{W}_{\mathrm{RTS}}$ d. $S_{l l}$ versus $\mathrm{W}_{\mathrm{RTS}}$. 

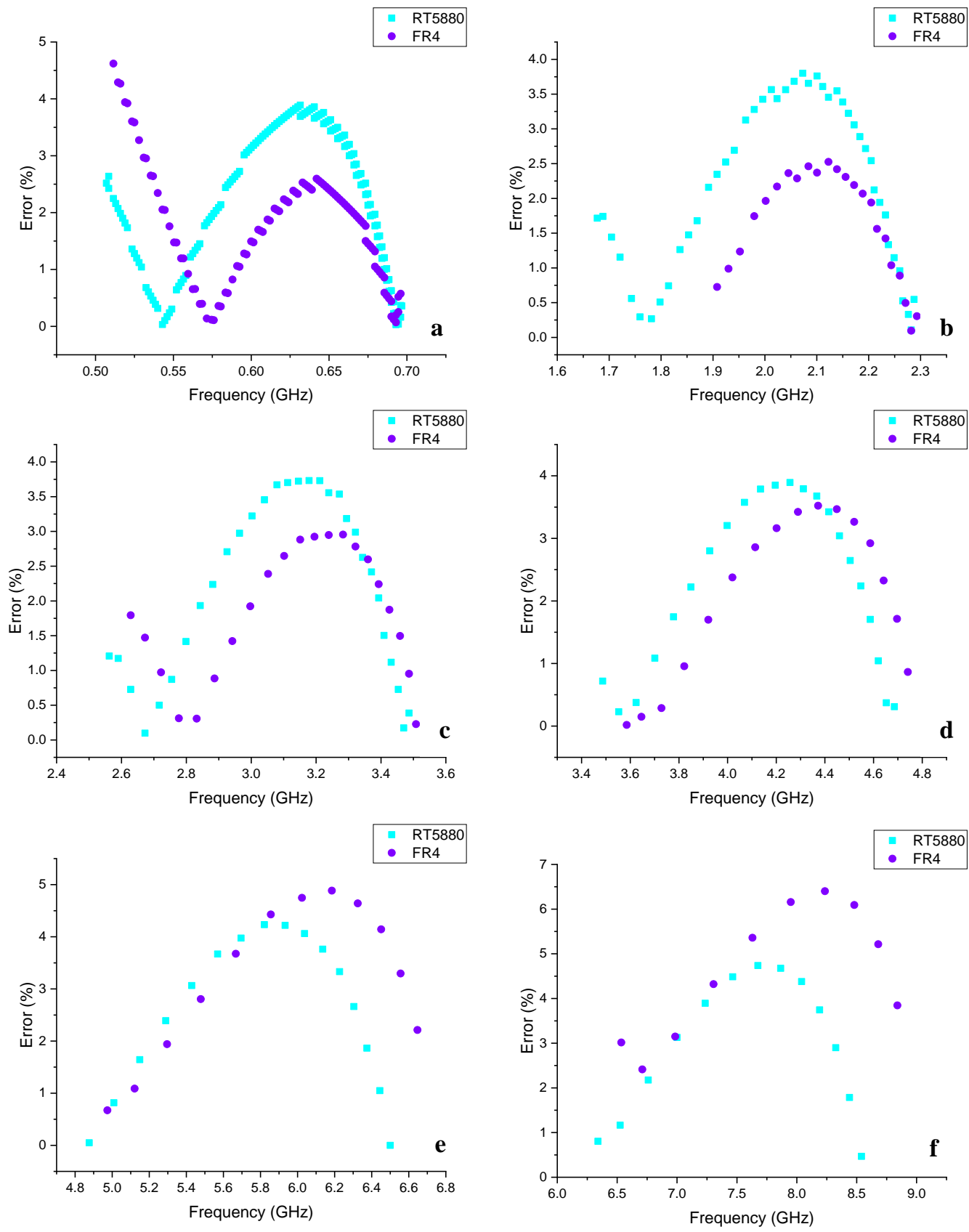

Figure 5. Percentage error values according to the simulation results of the developed formula for different frequencies, a. $f_{r}=700 \mathrm{MHz}$ b. $f_{r}=2300 \mathrm{MHz}$ c. $f_{r}=3500 \mathrm{MHz}$ d. $f_{r}=4700 \mathrm{MHz}$ e. $f_{r}=6500 \mathrm{MHz}$ f. $f_{r}=8500 \mathrm{MHz}$.

There are microstrip antennas with tuning stubs in the literature. The comparison of some of these with our study is given in Table 3. The general purpose of adding tuning stubs, as is known, is to adjust the antenna around a specific operating frequency. The typical features of the compared studies are that they have more complex geometries and require time-consuming simulations for each size variation. However, in this study, both the adjustment range has been extended, and an analytical model has been developed that gives approximately $2 \%$ accurate results. Thus, there is no need for repeated long simulation processes for each geometric value.

\section{CONCLUSIONS}

Patch antennas with rectangular tuning stubs operating at early phase $5 \mathrm{G}$ bands are designed and simulated. The effects on the radiation characteristics are analyzed by performing a multi-parameter analysis. It has been shown that the edge dimension variations of the stub directly affect the resonant frequency of the antenna and reflection coefficient levels. Also, for an optimal value of the stub width, the frequency shift amount and reflection coefficient levels are reasonable, 
1/3.5 of the patch width. After performing a detailed parametric analysis for the stub length, a design equation is proposed. This equation relates the antenna's resonant frequency to the stub length, with the condition of stub width having a definite optimal value.

\section{REFERENCES}

[1]. Du Plessis, M., and John Cloete. "Tuning stubs for microstrip-patch antennas.” IEEE Antennas and Propagation magazine 36.6 (1994): 52-56.

[2]. Erdil, Emre, et al. "Frequency tunable microstrip patch antenna using RF MEMS technology." IEEE transactions on antennas and propagation 55.4 (2007): 1193-1196.

[3]. Sheta, Abdel-Fattah, and Samir F. Mahmoud. "A widely tunable compact patch antenna." IEEE Antennas and Wireless Propagation Letters 7 (2008): 40-42.

[4]. Liu, L., and R. J. Langley. "Liquid crystal tunable microstrip patch antenna." Electronics Letters 44.20 (2008): 1179-1180.

[5]. Liang, Jing, and HY David Yang. "Microstrip patch antennas on tunable electromagnetic band-gap substrates." IEEE transactions on antennas and propagation 57.6 (2009): 1612-1617.

[6]. Sung, Y. "Axial Ratio-Tuned Circularly Polarized Square Patch Antenna with Long Stubs." International Journal of Antennas and Propagation 2018 (2018).

[7]. Hasan, Wanis Alfitouri, et al. "Design and fabrication of triple frequency microstrip patch antenna by attaching tuning stub element." 2016 10th International Conference on Telecommunication Systems Services and Applications (TSSA). IEEE, 2016.

[8]. Arya, Ashwini K., Rao Shahid Aziz, and Seong-Ook Park. "Planar ultra-wideband printed wide-slot antenna using fork-like tuning stub.” Electronics Letters 51.7 (2015): 550 551.

[9]. Bokhari, S. A., et al. "A small microstrip patch antenna with a convenient tuning option." IEEE Transactions on antennas and Propagation 44.11 (1996): 1521-1528.

[10]. 5G Americas, (2017). 5G Spectrum Recommendations. https://www.5gamericas.org/wp-content/uploads/2019/07/5 GA_5G_Spectrum_Recommendations_2017_FINAL.pdf (Accessed: 02.01.2020).

[11]. Lee, Juho, et al. "Spectrum for 5G: Global status, challenges, and enabling technologies." IEEE Communications Magazine 56.3 (2018): 12-18.

[12]. Global Mobile Suppliers Association, (2018). Spectrum for Terrestrial 5G Networks: Licensing Developments Worldwide. https://gsacom.com/paper/5gspectrum-terrestrial-networks/ (Accessed:02.01.2020).

[13]. RSPG19-036 FINAL, Strategic Roadmap Towards 5G For Europe, Radio Spectrum Policy Group of European Commission, Brussels, 2019.

[14]. Balanis, Constantine A. "Antenna Theory Analysis And Design', New Jersey John Willey \& Sons.” Inc, Publication (2005).

[15]. Joler, Miroslav, and Josko Kucan. "Impact of Slot Parameters on the Three Resonant Frequencies of a Rectangular Microstrip Antenna: Study of the impact of the slot length, width, and position." IEEE Antennas and Propagation Magazine 57.4 (2015): 48-63.

[16]. Computer Simulation Technology (CST) Microwave Studio, Framingham, MA, USA, 2017.

[17]. Ray, K. P., and Kumar, G. "Tuneable and dual-band circular microstrip antenna with stubs." IEEE transactions on antennas and propagation, 48(7), 1036-1039, (2000).

[18]. Karmakar, N. C., "Shorting strap tunable stacked patch PIFA." IEEE Transactions on Antennas and Propagation, 52(11), 2877-2884, (2004). 